\title{
Molecular-dynamics simulation of thin-film growth by energetic cluster impact
}

\author{
Hellmut Haberland \\ Freiburger Materialforschungszentrum und Fakultät für Physik, Universität Freiburg, H. Herderstrasse 3, \\ D-79104 Freiburg, Germany \\ Zinetulla Insepov \\ Kazakh Polytechnical Institute, Alma Ata, Kazakhstan \\ Michael Moseler \\ Freiburger Materialforschungszentrum und Fakultät für Physik, Universität Freiburg, H. Herderstrasse 3, \\ D-79104 Freiburg, Germany \\ (Received 30 August 1994)
}

\begin{abstract}
Langevin-molecular-dynamics simulations of thin-film growth by energetic cluster impact were carried out. The impact of a $\mathrm{Mo}_{1043}$ cluster on a $\mathrm{Mo}(001)$ surface was studied for impact energies of $0.1,1$, and $10 \mathrm{eV} /$ atom using the Finnis-Sinclair many-body potential. The characteristics of the collision range from a soft touchdown at $0.1 \mathrm{eV} /$ atom, over a flattening collision at $1 \mathrm{eV} / \mathrm{atom}$, to a meteoric impact at $10 \mathrm{eV} /$ atom. The highest energy impact creates a pressure of about 100 $\mathrm{GPa}$ in the impact zone and sends a strong shock wave into the material. The cluster temperature reaches a maximum of $596 \mathrm{~K}$ for $0.1 \mathrm{eV} /$ atom, $1799 \mathrm{~K}$ for $1 \mathrm{eV} /$ atom, and $6607 \mathrm{~K}$ for $10 \mathrm{eV} /$ atom during the first ps after the touchdown. For energies of 1 and $10 \mathrm{eV} /$ atom the cluster recrystallizes after $20 \mathrm{ps}$. The consecutive collision of $50 \mathrm{Mo}_{1043}$ clusters with a $\mathrm{Mo}(001)$ surface at $T=300 \mathrm{~K}$ was simulated for the three impact energies. The formation of a porous film is calculated for clusters impinging with low kinetic energy, while for the clusters with the highest energy a dense mirrorlike film is obtained, in good agreement with experiment.
\end{abstract}

\section{INTRODUCTION}

An experimental technique was recently described that uses the impact of energetic clusters for thin-film formation. ${ }^{1-3}$ Charged metal clusters ( $\mathrm{Al}, \mathrm{Co}, \mathrm{Cu}, \mathrm{Fe}, \mathrm{Mo}$, or $\mathrm{Ti}$ ) containing one charge and $10^{3}$ to $10^{4}$ atoms are electrically accelerated and deposited on a surface. The experimentally observed thin-film morphology depends strongly on the kinetic energy of the impinging cluster. For slow clusters, with an impact energy of about $0.1 \mathrm{eV}$ per atom, the resulting films are black or colored and can easily be wiped off. At a medium energy of about $1 \mathrm{eV}$ per atom the films are colored with a better adhesion. In the high-energy regime ( $10 \mathrm{eV}$ per atom), strongly adhering, mirrorlike films appear. The name energetic cluster impact (ECI) was proposed for this method of thin-film formation. ${ }^{1-3}$

Several attempts have been made earlier to understand thin-film formation by clusters either by moleculardynamics (MD) (Refs. 4-10) or by Monte Carlo methods. ${ }^{11,12}$ Most of the authors studied the collision of a single cluster with an ideal surface. This allows one to study the cluster-surface interaction but is not sufficient to understand thin-film morphology in detail. Only a few authors have investigated the growth of the film by more than one cluster. Müller ${ }^{4}$ published a two-dimensional MD simulation using a Lennard-Jones potential, which is not adequate for metals. Also the restrictions to two dimensions might be too simplifying. Kwon et al. ${ }^{5}$ modeled the growth of Si films by small Si clusters $(N=33)$. Though more realistic, their findings are not applicable to metallic ECI films.

A three-dimensional simulation of an ECI grown film using metal clusters containing around 1000 atoms was started, in order to simulate the experimental conditions realistically. In an earlier MD study ${ }^{9}$ the impact of one single $\mathrm{Mo}_{1024}$ cluster impinging on a Mo(001) surface was simulated. We again used molybdenum for the calculations presented here. Results on copper will be published elsewhere. ${ }^{13}$

\section{SIMULATION MODEL}

The evolution of the atoms in time and space was determined by the numerical solution of the classical equations of motion which were integrated by the usual Euler algorithm. For metallic systems the embedded atom method (EAM) potential provides a reasonably accurate description of interatomic forces. ${ }^{14,15}$ EAM is based on local density functional theory, which asserts that the energy of a solid can be written as a unique functional of the electron density, which is assumed to be the local density at each atomic site. The local electronic density at the position of an atom is approximated by the superposition of the atomic electronic densities of the surrounding atoms. Therefore, the total energy of an arbitrary arrangement of $N$ atoms is given by a sum of the energies $E_{i}$ of the atoms,

$$
E_{\mathrm{tot}}=\sum_{i=1}^{N} E_{i}
$$


which is written as a sum of two terms,

$$
E_{i}=F\left(\rho_{i}\right)+\frac{1}{2} \sum_{j \neq i} \phi\left(r_{i j}\right) .
$$

The embedding energy $F\left(\rho_{i}\right)$ depends on the local electronic charge density at the position of atom $i$. This density is obtained by adding up the contributions $f\left(r_{i j}\right)$ of the other atoms

$$
\rho_{i}=\sum_{j(\neq i)} f\left(r_{i j}\right)
$$

where $r_{i j}$ is the distance between atom $i$ and $j$. The second part in (2) belongs to the core-core repulsion, which is given by the pair potential $\phi\left(r_{i j}\right)$.

Finnis and Sinclair ${ }^{16}$ used tight-binding theory to develop a many-body potential for bcc metals, which turned out to be mathematically equivalent to the EAM. They determined the EAM functions by a fit to the equilibrium lattice constant, the bulk modulus, the elastic constants, and the heat of sublimation. We used the Finnis-Sinclair potential for molybdenum. In order to predict highenergetic events correctly the improvement proposed by Ackland and Thetford ${ }^{17}$ was employed.

The number of surface atoms that can be taken into account is limited by our present hardware (HP735) to a few $10^{4}$. Thus, only atoms in a small part of the surface (the primary zone ${ }^{18}$ ) can be simulated by MD. The rest of the atoms (those in the secondary zone) are kept at fixed positions. We choose a half sphere around the impact zone as the primary zone (see Fig. 1). The outer two atomic layers of the primary zone were declared to be edge atoms. The primary zone was surrounded by a zone of fixed atoms, representing the secondary zone. The two layers of edge atoms are important, as they have to simulate the heat conductivity of the solid. Also the strong shock wave resulting from the impact of an energetic cluster has to be damped out by the edge atoms. The specification of an unfavorable boundary condition there would lead to a reflection of the shock wave by the rigid secondary zone. Adelmann and Doll ${ }^{19}$ used the harmonic approximation for the interatomic potentials in

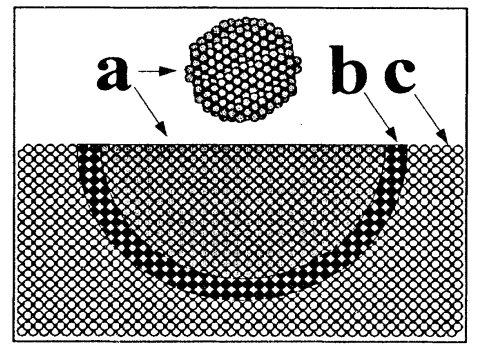

FIG. 1. Atoms in the cluster and the surface are partitioned into three zones: (a) atoms in the cluster and around the impact area are treated by molecular dynamics. The edge atoms in zone (b) are treated by Langevin molecular dynamics in order to simulate the heat conductivity of the semi-infinite bulk. Atoms in part (c) are fixed, and supply only an outer frame. Up to 12000 moveable atoms have been treated. the solid and Debye theory to show that the atoms of the active zone obey the Langevin equation

$$
\ddot{\mathbf{r}_{k}}=\frac{1}{m_{k}} \mathbf{F}_{k}\left(\mathbf{r}_{1}, \ldots, \mathbf{r}_{N}\right)-\gamma_{k} \dot{\mathbf{r}}_{k}+\sqrt{\frac{2 \gamma_{k} k T}{m_{k}}} \boldsymbol{\eta}_{k},
$$

where $\mathbf{r}_{k}$ is the position vector and $m_{k}$ the mass of the $k$ th atom, $\mathbf{F}_{k}$ the Finnis-Sinclair force, $k$ the Boltzmann constant, $T$ the temperature of the solid, and $\boldsymbol{\eta}_{k}$ a vector of Gaussian random numbers with $\left(\left\langle\boldsymbol{\eta}_{m}(t) \boldsymbol{\eta}_{k}\left(t^{\prime}\right)^{T}\right\rangle\right)_{i j}=$ $\delta\left(t-t^{\prime}\right) \delta_{i j} \delta_{m k}$. The friction coefficient $\gamma_{k}$ is zero for the inner atoms of the primary zone and for cluster atoms (usual MD) and $\gamma_{k}=\frac{\pi}{6} \omega_{D}$ ( $\omega_{D}$ : Debye frequency) for edge atoms of the primary zone (Langevin MD). The choice of $\gamma_{k}$ ensures that the long time energy transfer into the bulk is given correctly. The second and the third term in (4) are related by the second fluctuationdissipation theorem. ${ }^{19}$ Thus, the edge atoms form a heat bath for the active zone.

The surface was simulated by $80 \times 30 \times 18$ bcc cells, containing altogether 86400 atoms. The number of particles in the primary zone depended on the impact energy (up to 12000 for $10 \mathrm{eV} /$ atom). The point of impact was chosen randomly. Cyclical boundary conditions were used for the directions tangential to the surface. A spherical cutout off a bcc lattice formed a cluster of 1043 atoms. The cluster was rotated by three random Euler angles and positioned at a distance of two lattice constants above the surface. Each new cluster defined its own new primary zone. Before starting the cluster with a velocity vertical to the surface, cluster and primary zone were thermalized for 100 time steps at $T=300 \mathrm{~K}$. The equations of motion were integrated with a time step of 1 to 5 fs ( $\frac{1}{20}$ of the period obtained from the Debye frequency). The motion of the system was simulated for $20 \mathrm{ps}$, holding the temperature of the edge atoms on $T=300 \mathrm{~K}$. Afterwards cluster and primary zone were quenched to $0 \mathrm{~K}$ and the next cluster was started.

The collision of a cluster with a surface is a highly dynamical process. Nevertheless we are interested in equilibrium properties such as temperature or pressure of the system. To define the local temperature of a nonequilibrium system we subtracted from the kinetic energy of an atom the kinetic energy of the collective movement of the atom and his next neighbors. The local temperature was obtained from this difference using the equipartition theorem of statistical mechanics. Egami and Srolowitz ${ }^{20}$ generalized the pressure of equilibrium statistical mechanics to a local pressure for nonequilibrium systems of atoms interacting with pair potentials. We extended their approach to EAM many-body potentials, obtaining the local static pressure at the location of atom $i$,

$$
\begin{aligned}
p_{s}\left(\mathbf{r}_{i}\right)= & -\frac{1}{3}\left[n ( \mathbf { r } _ { i } ) \sum _ { j ( \neq i ) } \left(\frac{1}{2} \phi^{\prime}\left(r_{i j}\right)\right.\right. \\
& \left.\left.+F^{\prime}\left(\rho_{i}\right) f^{\prime}\left(r_{i j}\right)\right) r_{i j}\right] .
\end{aligned}
$$

Here $n$ denotes the local density (as defined in Ref. 8). The local static pressure $p_{s}\left(\mathbf{r}_{i}\right)$ given at the atom sites 


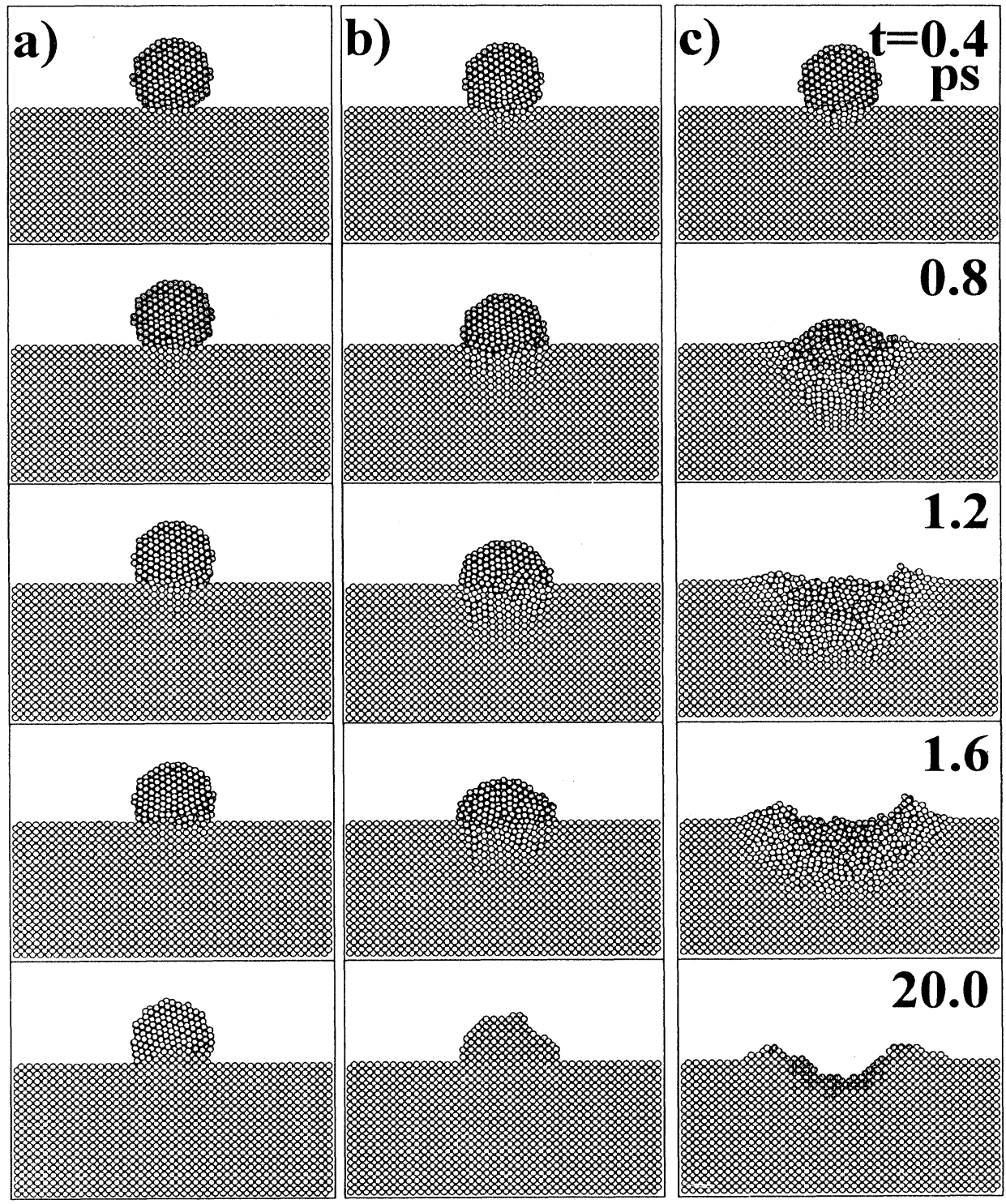

FIG. 2. A $\mathrm{Mo}_{1043}$ cluster with (a) $0.1 \mathrm{eV}$, (b) $1 \mathrm{eV}$, and (c) $10 \mathrm{eV}$ kinetic energy per atom impinges on a Mo(001) surface. A three-atom-deep cut through the surface is shown. Note the shock wave propagating into the surface for the 10-eV collision.

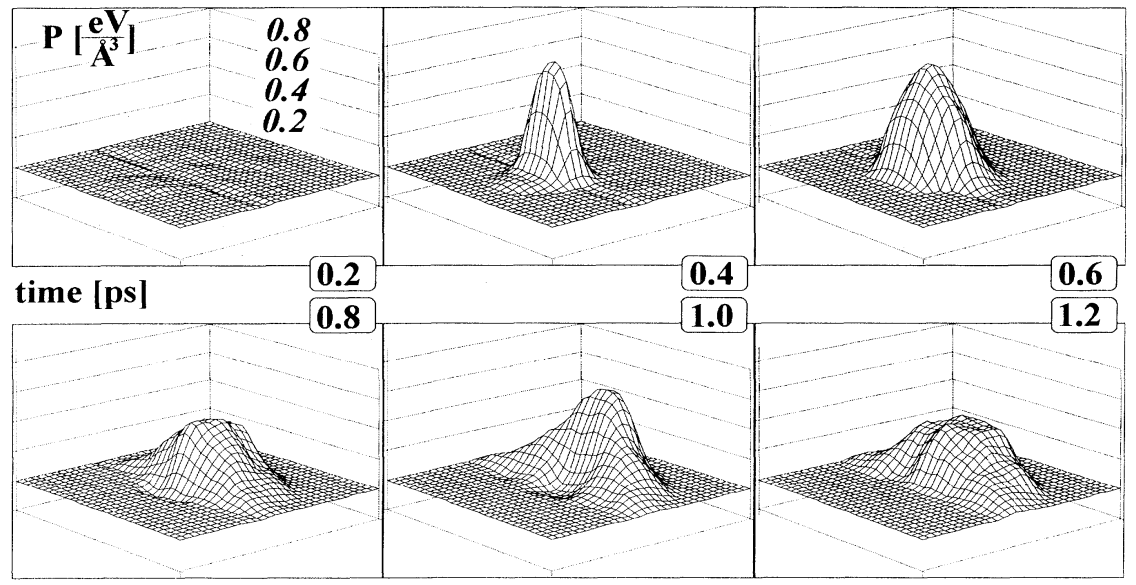

FIG. 3. The local pressure (on a plane through the impact mark, perpendicular to the surface) for the collision with 10 $\mathrm{eV}$ kinetic energy per atom. A shock wave is moving into the solid. 

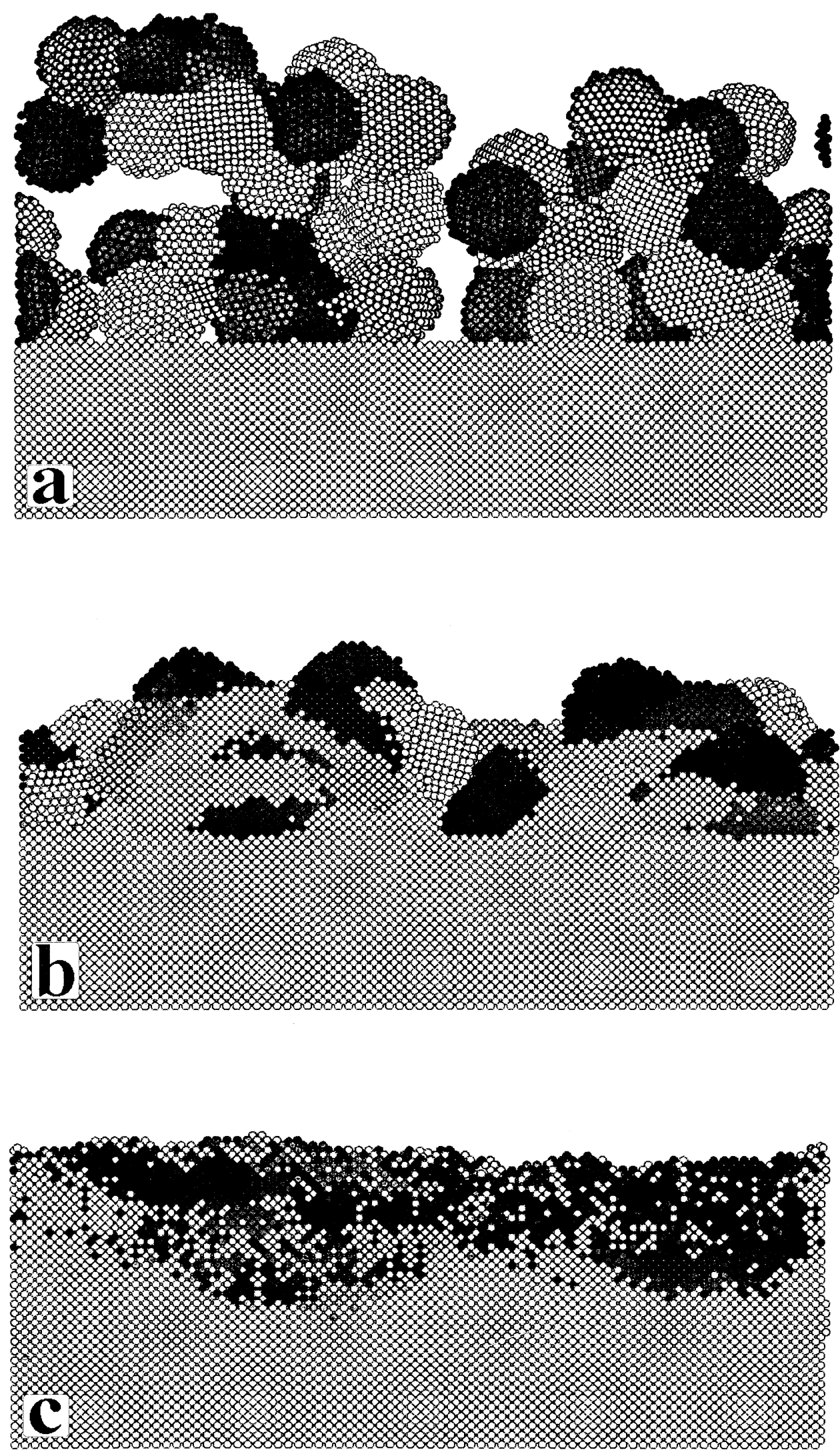

FIG. 4. ECI films formed by $\mathrm{Mo}_{1043}$ clusters with (a) $0.1 \mathrm{eV}$ (b) $1 \mathrm{eV}$ and (c) $10 \mathrm{eV}$ kinetic energy per atom. 
was continued by a weighted average onto the whole space and a second term was added to account for the local temperature $T(\mathbf{r})$ (obtained by the same weighted averaging),

$$
\begin{aligned}
P(\mathbf{r}) & =\sum_{i=1}^{N} g\left(\left|\mathbf{r}-\mathbf{r}_{i}\right|\right) p_{s}\left(\mathbf{r}_{i}\right)+n(\mathbf{r}) k T(\mathbf{r}), \\
\sum_{i=1}^{N} g\left(\left|\mathbf{r}-\mathbf{r}_{i}\right|\right) & =1
\end{aligned}
$$

We chose the function $g$ to be a Gaussian with an appropriate standard deviation ( $3 \AA$ ). It should be stressed that there is some arbitrariness in defining such local quantities. Nevertheless they are useful for the qualitative understanding of a nonequilibrium process.

\section{RESULTS}

\section{A. Single cluster impact}

As a preliminary step, the collision of a single Mo cluster with a Mo(001) surface was examined. The time step was chosen to be 1 fs. Figure 2 shows the dynamics of the deposition process. The outcome of the collision ranges from a soft touchdown at $0.1 \mathrm{eV} /$ atom, over a strong flattening of the cluster at $1 \mathrm{eV} /$ atom, to a meteoric impact at $10 \mathrm{eV} /$ atom. Even for the highest impact energy no ejection of atoms was observed. The temperature of the cluster was calculated for each time step. The maximum temperature was 596,1799 , and $6607 \mathrm{~K}$ for the three impact energies, respectively. Thinking in equilibrium concepts, this would indicate a melting of the cluster for the $10-\mathrm{eV} /$ atom collision; but the equilibrium concept is not appropriate in the first few ps of the $10-\mathrm{eV} /$ atom collision; it will become valid only at later times.

The cluster is completely torn apart for the 10$\mathrm{eV} /$ atom collision, and the final distribution of atoms is a highly nonequilibrium one. A strong shock wave (see Fig. 3) is generated. In the experiment this "nano-shock wave" 1,8 runs into the surface and is absorbed there. In the simulations, the shell of edge atoms has to perform this task, as discussed above. A peak pressure of about $100 \mathrm{GPa}$ (1 Mbar) is generated at this impact.

\section{B. Simulation of film formation}

It was observed that the qualitative outcome of a single impact did not change strongly on increasing the time step to $5 \mathrm{fs}$. This increased time step reduces the computer time sufficiently so that a simulation of film growth became possible.

The resulting film microstructure can be seen by plotting either the final coordinates of the atoms (Fig. 4) or by looking at the film density in the direction vertical to the surface (Fig. 5). The simulations for the three impact energies give three different pictures for the film morphology. The slow clusters $(0.1 \mathrm{eV} /$ atom $)$ do not break apart, but are gently stocked one above the other. The dendritelike film has a very large surface and consequently appears black. Little to no intermixing of cluster with surface atoms occurs, so it would be easy to mechanically remove the film. Moreover, the film possesses less than one half of the bulk density.

Clusters with medium impact energy ( $1 \mathrm{eV} /$ atom $)$ grow to a much denser, epitaxial film with only a few cavities (Fig. 6). The biggest cavity had about the size of a cluster. Cluster and surface atoms are mixed within a distance of one lattice constant (Fig. 5). This leads to a better adhesion compared to the lower-energy collision. The density reaches $80 \%$ of the bulk.

The 10-eV/atom clusters form a dense epitaxial film, which has nearly the bulk density. Only few and small voids exist (Fig. 6). The original shape of a single cluster is hardly perceptible, because its form was destroyed by the following cluster impacts. Strong intermixing between cluster and surface material is observable, resulting in good adhesion.

We performed an additional simulation of thin-film growth with a slightly different surface geometry and another series of random numbers for the points of impact. As we observed the same film structures, we conclude that the initial conditions have no essential influence on our results.

\section{DISCUSSION}

The results show that a three-dimensional moleculardynamics study of thin-film formation is possible, even if a realistic many-body potential is used.

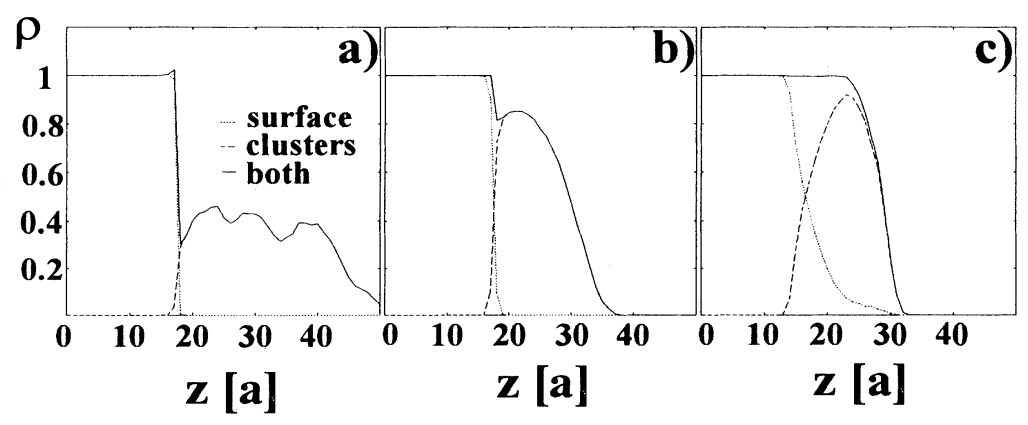

FIG. 5. The film-density (smoothed by a moving average) in the direction perpendicular to the surface for (a) $0.1 \mathrm{eV}$ (b) $1 \mathrm{eV}$ and (c) $10 \mathrm{eV}$ kinetic energy per cluster atom. The equilibrium density of bcc Mo was set to 1. The distance is measured in multiples of the equilibrium lattice constant corrections. 


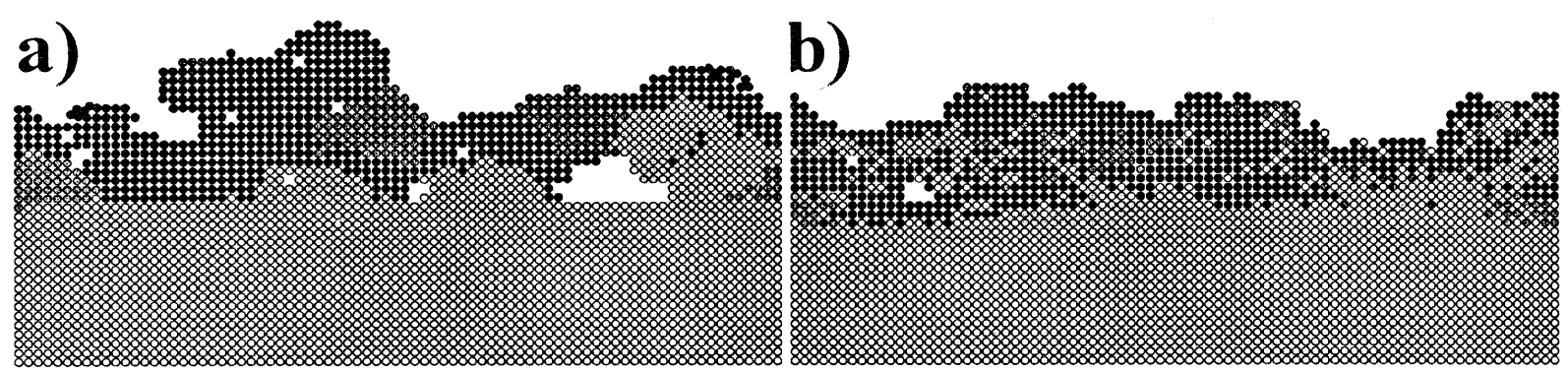

FIG. 6. Cavities in the films for (a) $1 \mathrm{eV}$ and (b) $10 \mathrm{eV}$ kinetic energy per atom. A one-atom-deep cut through the biggest cavities is shown.

\section{A. Comparison with experiment}

The simulations give a microscopic picture of the growth process and of the resulting thin films produced. The calculated dependence of the film microstructure on the energy of the molybdenum clusters is in qualitative agreement with experiment. We expect the thin-film formation to be qualitatively similar for other metals. Experimentally at least, no large differences are observed, when changing from $\mathrm{Mo}$ to $\mathrm{Al}, \mathrm{Cu}, \mathrm{Ti}$, or $\mathrm{TiN}$.

In the experiment ${ }^{1-3}$ the substrate is irradiated by a flux of about $10^{12}$ clusters $/ \mathrm{cm}^{2} \mathrm{~s}$. This leads to a growth rate of about one monolayer/s. Stated otherwise, each spot on the surface or its near surrounding is hit by about one cluster every second. Considerable surface diffusion and/or nondiffusional solid-on-solid settling ${ }^{10}$ is possible during this time if the temperature is high enough. These long time scales cannot be simulated by molecular dynamics. The simulations reported here have been extended only to $20 \mathrm{ps,}$ which is about 11 orders of magnitude less. Therefore, any effect of a long term surface mobility is far beyond the possibilities of present day MD calculations.

The experiment and the simulation have been performed for a surface temperature of $300 \mathrm{~K}$, which is only about $10 \%$ of the bulk Mo melting point. For so low a temperature the mobility of the atoms and clusters on the surface can be neglected. ${ }^{10}$ Therefore, a moleculardynamics study of film growth at room temperature by many clusters does provide a valid explanation of the experimentally observed film microstructure.

\section{B. Limitations and improvements}

The EAM potentials are obtained from equilibrium properties of the solid. It is unknown if they can describe dynamical processes as well. For example, the electronic excitation by the energetic ions is disregarded. This is the reason why EAM molybdenum possesses a thermal heat conductivity that is roughly a factor of 100 smaller than that of real Mo. Furthermore, the actual surface will be stiffer for highly dynamical processes, because of the additional damping of the cluster atoms by collisions with the electrons of the surface. This may imply some errors for the film morphology with $10 \mathrm{eV} /$ atom impact energy. Further work to investigate such effects is in progress.

The maximum size of cavities, which would be observable in our simulation, is of the order of the simulated surface piece. However, cavities caused by statistical fluctuation in the distribution of the impact marks could be much bigger. Of course, such effects are inaccessible for molecular dynamics. In comparison with Müller's work ${ }^{4}$ it should be noted, that three-dimensional effects play only a little role in the qualitative description of the films. Apart from the ejection of cluster atoms in Müller's highenergetic collision there were no important differences. But for a quantitative investigation (e.g., comparing calculated with measured film densities) the use of realistic models is essential. In the future we will elaborate a molecular-dynamics model for $\mathrm{Cu}$ that incorporates a much better potential (EAM works best for fcc metals) and consider the electron-ion coupling.

\section{ACKNOWLEDGMENT}

The research was partially supported by the BriteEuram program, Project No. BRE2-0534 of the Commission of the European Communities.
${ }^{1}$ H. Haberland, M. Karrais, M. Mall, and Y. Thurner, J. Vac. Sci. Technol. A 10, 3266 (1992).

${ }^{2}$ H. Haberland, M. Mall, M. Moseler, Y. Quiang, Th. Reiners, and Y. Thurner, Nucl. Instrum. Methods Phys. Res. Sect. B 80/81, 1320 (1993).

${ }^{3}$ H. Haberland, M. Mall, M. Moseler, Y. Qiang, Th. Reiners, and Y. Thurner, J. Vac. Sci. Technol. A 12, 2925 (1994).

${ }^{4}$ K. Müller, J. Appl. Phys. 61, 2516 (1987).
${ }^{5}$ I. Kwon, R. Biswas, G.S. Grest, and C.M. Soukoulis, Phys. Rev. B 41, 3678 (1990).

${ }^{6}$ H. Hsieh, R.S. Averback, H. Sellers, and C.P. Flynn, Phys. Rev. B 45, 4417 (1992).

${ }^{7}$ J.D. Pelletier, M.H. Shapira, and T.A. Tombrello, Nucl. Instrum. Methods Phys. Res. Sect. B 67, 256 (1992).

${ }^{8}$ C.L. Cleveland and U. Landmann, Science 257, 355 (1992).

${ }^{9}$ H. Haberland, Z. Insepov, and M. Moseler, Z. Phys. D 26, 
229 (1993).

${ }^{10}$ W.D. Luedtke and Uzi Landman, Phys. Rev. Lett. 73, 569 (1994).

${ }^{11}$ Y. Yamamura, I. Yamada, and T. Takagi, Nucl. Instrum. Methods Phys. Res. Sect. B 37/38, 902 (1989).

${ }^{12}$ Y. Yamamura, Nucl. Instrum. Methods Phys. Res. Sect. B 45, 707 (1990).

${ }^{13} \mathrm{M}$. Moseler and H. Haberland (unpublished).

${ }^{14}$ M.S. Daw and M.I. Baskes, Phys. Rev. Lett. 50, 1285 (1983).
${ }^{15}$ M.S. Daw and M.I. Baskes, Phys. Rev. B 29, 6443 (1984).

${ }^{16}$ M.W. Finnis and J.E. Sinclair, Philos. Mag. A 50, 54 (1984).

${ }^{17}$ G.J. Ackland and R. Thetford, Philos. Mag. A 56, 15 (1987).

${ }^{18}$ A.E. DePriesto and H. Metiu, J. Chem. Phys. 90, 1229 (1989).

${ }^{19}$ S.A. Adelmann and J.D. Doll, J. Chem. Phys. 64, 2375 (1976); 86, 4885 (1987).

${ }^{20}$ T. Egami and D. Srolowitz, J. Phys. 12, 2141 (1982). 